\title{
Material Properties Estimation of Layered Soft Tissue Based on MR Observation and Iterative FE Simulation
}

\author{
Mitsunori Tada ${ }^{1,2}$, Noritaka Nagai $^{3}$, and Takashi Maeno ${ }^{3}$ \\ 1 Digital Human Research Center, \\ National Institute of Advanced Industrial Science and Technology, \\ 2-41-6, Aomi, Koto-ku, Tokyo 135-0064, Japan \\ m.tada@aist.go.jp \\ 2 CREST, Japan Science and Technology Agency \\ ${ }^{3}$ Keio University
}

\begin{abstract}
In order to calculate deformation of soft tissue under arbitrary loading conditions, we have to take both non-linear material characteristics and subcutaneous structures into considerations. The estimation method of material properties presented in this paper accounts for these issues. It employs a compression test inside MRI in order to visualize deformation of hypodermic layered structure of living tissue, and an FE model of the compressed tissue in which non-linear material model is assigned. The FE analysis is iterated with updated material constant until the difference between the displacement field observed from MR images and calculated by FEM is minimized. The presented method has been applied to a 3-layered silicon rubber phantom. The results show the excellent performance of our method. The accuracy of the estimation is better than $15 \%$, and the reproducibility of the deformation is better than $0.4 \mathrm{~mm}$ even for an FE analysis with different boundary condition.
\end{abstract}

\section{Introduction}

The rapid progress in computational power and algorithm enable us to carry out finite element (FE) analysis of massive mechanical structures. This technique is being applied to the structural simulation of human body that lead to successful stress/strain analysis of hard tissue such as bone.

In surgical training system and computer assisted diagnosis system, precise $\mathrm{FE}$ analysis of soft tissue is required in order to predict the response of the tissue under arbitrary loading conditions. Mechanical characterization of living soft tissue is thus one of the key technologies in such medical systems.

Many methodologies have been proposed for estimating mechanical properties of living soft tissue. One intuitive method is to indent the surface of the tissue [12]. Material property is then obtained from the relation between the indentation depth and the reaction force. Although this method is simple and applicable to the entire surface of human body, they have two fatal disadvantages. They cannot take large deformation and subcutaneous structure into considerations as is obvious from the principle. 
Recent progress in diagnostic imaging modalities allows us to develop new elasticity imaging techniques. Magnetic resonance elastography (MRE) is one of the promising method 3/4. It visualizes strain waves that propagate within soft tissue by using MRI. We can estimate distribution of the stiffness even for a subcutaneous tissue since the wavelength visualized by MRE is proportional to the stiffness. However, several problems arise when applying this technique to a practical application. Damping and reflection of the strain waves cause artifacts in MR image, and thus they lead to error in the stiffness estimation.

Quasi-static MRE is another elasticity imaging method [5]. It employs a constitutive equation of linear elasticity to reconstruct the stiffness distribution from the strain field visualized also by MRI. It was found, however, that in this method, since the constitutive equation assumes small deformation, non-linear characteristics of soft tissue caused by large deformation cannot be taken into accounts.

In order to calculate deformation of soft tissue under arbitrary loading conditions, we have to take both non-linear material characteristics and subcutaneous structures into considerations. The estimation method of material properties presented in this paper accounts for these issues. It employs a compression test inside MRI in order to visualize deformation of hypodermic layered structure of living tissue, and an FE model of the compressed tissue in which non-linear material model is assigned. The FE analysis is iterated with updated material constant until the difference between the displacement field observed from MR images and calculated by FEM is minimized.

Different from similar approaches presented in the literature [6], we employ an MR-compatible optical force sensor [7] in order to determine the strict boundary conditions for the FE analysis. Furthermore, the extended Kalman filter used in the iterative optimization help us to achieve fast convergence to the final estimate. The presented method has been applied to a 3-layered silicon rubber phantom. The results show the excellent performance of our method. The accuracy of the estimation is better than $15 \%$, and the reproducibility of the deformation is better than $0.4 \mathrm{~mm}$ even for an $\mathrm{FE}$ analysis with different boundary condition.

\section{Method and Implementation}

\subsection{Method Overview}

Our method for material properties estimation involves four steps.

MR compression test: Compress the tissue inside MRI in order to visualize deformation of the subcutaneous structure. The reaction force is simultaneously measured by using an MR-compatible force sensor [7.

MR image processing: Extract the displacement field within the tissue by applying an image registration technique to the obtained MR images.

FE mesh generation: Generate a finite element model of the subcutaneous structure from the pre-compression MR image. The same boundary conditions as the MR compression test are given to the FE model. 
Iterative FE analysis: Assign the initial estimate of the material constant to the model, and repeat the FE analysis with updated material constant until the difference between the displacement field observed from MR images and calculated by FEM is minimized (see Section 2.2 for detail).

This method has distinct advantages over the conventional approaches [1 2 3 4 45. Firstly, both non-linear characteristics and subcutaneous structure are incorporated at a time. Secondly and equally important for medical applications, the reproducibility of the $\mathrm{FE}$ analysis in which the estimated material properties are assigned is guaranteed, since they are determined so that the result of the $\mathrm{FE}$ analysis is adapted to the observed deformation.

\subsection{Implementation of the Iterative FE Analysis}

To be precise, the iterative $\mathrm{FE}$ analysis is a minimization process of a disparity function $D$ defined by Equation (1),

$$
D(\boldsymbol{m})=\sum_{i}\left(d_{i}^{M R I}-d_{i}^{F E M}(\boldsymbol{m})\right)^{2}
$$

where, $\boldsymbol{m}$ is the material constant, $d_{i}^{M R I}$ is the observed displacement, $d_{i}^{F E M}(\boldsymbol{m})$ is the calculated displacement when the material constant is $\boldsymbol{m}$, and $i$ is an index of the corresponding point between the MR image and the FE model.

An extended Kalman filter (EKF) is employed for the minimization of the disparity function. In the case of this implementation, the measurement equation of the EKF is the FE analysis itself, and material constant $\boldsymbol{m}$ does not change during the time update phase of the EKF (a steady condition is given to the state equation). Functional capability of this Kalman filter is therefore equivalent to that of non-linear optimization algorithms such as Levenberg-Marquadt method.

As formulated in Equation (2), Kalman gain $\boldsymbol{K}_{k}$ at step $k$ is calculated from the error covariance $\boldsymbol{P}_{k-1}$, the measurement noise covariance $\boldsymbol{R}_{k}$ and the measurement jacobian $\boldsymbol{H}_{k}$.

$$
\boldsymbol{K}_{k}=\boldsymbol{P}_{k-1} \boldsymbol{H}_{k}^{T}\left(\boldsymbol{H}_{k} \boldsymbol{P}_{k-1} \boldsymbol{H}_{k}^{T}+\boldsymbol{R}_{k}\right)^{-1}
$$

The measurement jacobian $\boldsymbol{H}_{k}$ is approximated by a numerical difference of the forward FE analyses as given by Equation (3),

$$
H_{i j}=\frac{\partial d_{i}}{\partial m_{j}}=\frac{d_{i}^{F E M}(\boldsymbol{m}+d \boldsymbol{m})-d_{i}^{F E M}(\boldsymbol{m})}{d m_{j}}
$$

where $d \boldsymbol{m}$ is the minute increment of the material constant that have $d m_{j}$ at the $j$-th element and 0 at the rests. Thus, if $\boldsymbol{m}$ contains $n$ variables, $n+1$ times $\mathrm{FE}$ analyses are required in total to compute the measurement jacobian. The time update and the measurement update process of the EKF is iterated until the disparity function $D(\boldsymbol{m})$ is minimized. This procedure is implemented on the commercial FE solver, MSC Marc2003, by using a programming language Python. 


\section{Material Model}

\subsection{Silicon Rubber Phantom}

The estimation method given in Section 2 is applied to a 3-layered silicon rubber phantom. The dimension of this phantom is $12 \mathrm{~mm}$ in both width and height, and $70 \mathrm{~mm}$ in depth. It has the same 3-layered structure along the longitudinal direction. Thus, mechanical behavior at the center section of this phantom is given by a two-dimensional plane strain model, if the boundary conditions are also identical along this direction.

The material constant of each layer is estimated by a uni-axial compression test for reference, and by the proposed method for validation. The detail description of the uni-axial compression test is given in Section 4.1 The results of the estimation by the presented method are given in Section 4.2 to 4.4

\subsection{Material Model}

To deal with non-linear characteristics of the silicon rubber, first-term Ogden model is employed [8]. It is one type of a hyperelastic model widely used in the analysis of rubber-like material and soft tissue [9]. As shown in Equation (4), the nominal stress $\sigma$ is formulated by a function of the nominal strain $\varepsilon$ and two material constants, $\mu$ and $\alpha$, in the case of a uni-axial compression/tension.

$$
\sigma=\mu\left((\varepsilon+1)^{\alpha-1}-(\varepsilon+1)^{-\frac{\alpha}{2}-1}\right)
$$

Shown in Fig. 1 is the relation between the nominal strain $\varepsilon$ and the normalized nominal stress $\sigma / E$ (nominal stress divided by the Young's modulus $E$ ) for different $\alpha$. As is obvious from this figure, the stress-strain curves are almost identical when the strain is within the range of -0.5 to 0.25 that shows the redundancy between the two material parameters. This redundancy inhibits us

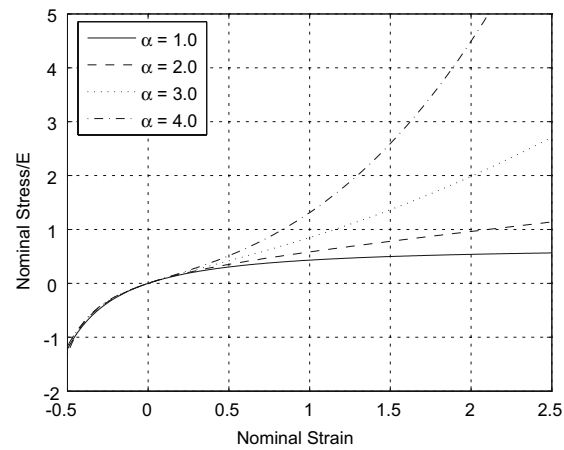

Fig. 1. Stress-strain diagrams of the first-term ogden model for different $\alpha$

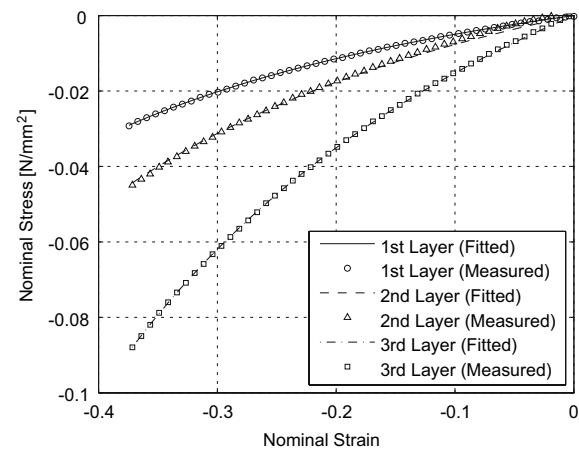

Fig. 2. Stress-strain diagrams of the silicon rubber in each layer 
from estimating them uniquely by a compression test. The material constant $\alpha$ is therefore fixed to 1.4 referring to the conventional solution [10]. The material model used in this research is finally given by Equation (5).

$$
\sigma=\mu\left((\varepsilon+1)^{0.4}-(\varepsilon+1)^{-1.7}\right)
$$

The simplified Ogden model contains only one material constant to be estimated. The material constant $\boldsymbol{m}$ in Equation (1) is thus given by $\left(\mu_{1 s t}, \mu_{2 n d}, \mu_{3 r d}\right)^{T}$, where subscript means the position of the rubber in the layered structure.

\section{Experiment}

\subsection{Uni-axial Compression Test}

Three cylindrical silicon rubbers that have $8 \mathrm{~mm}$ in height and diameter are prepared for the uni-axial compression test. Each rubber has the same material property as each layer of the phantom. Both end surfaces of the cylindrical rubbers are lubed by a silicon oil to achieve ideal uni-axial compression. They are compressed by a linear stage with the indentation speed of $0.1 \mathrm{~mm} / \mathrm{sec}$ until the indentation depth becomes $3.0 \mathrm{~mm}$.

Figure. 2 shows the stress-strain diagrams of the cylindrical rubbers. The material constant $\mu$ is identified by fitting Equation (5) to each diagram. The results of the identification are shown in the second column of Table 1 .

\subsection{MR Compression Test}

Shown in Fig. 3.(a) and (b) are the MR images of the center section during the compression test with a flat indenter and a cylindrical indenter, respectively.
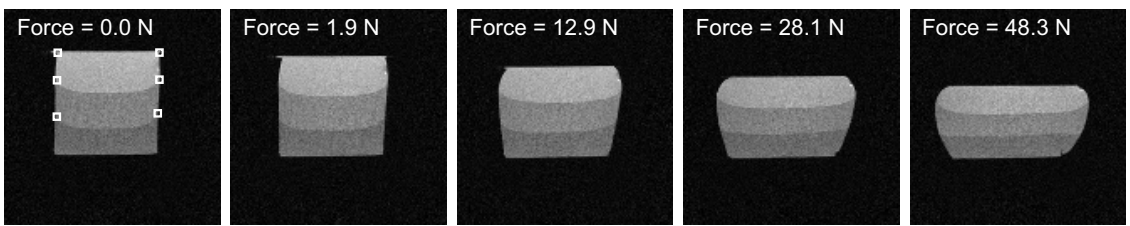

(a) Compression test by a flat indentor
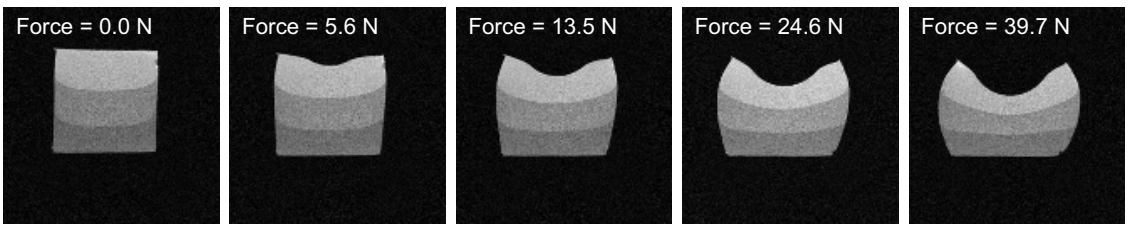

(b) Compression test by a cylindrical indentor

Fig. 3. Results of the MR compression test 
All images have a resolution of $256 \times 256$ pixels and a pixel size of $0.098 \times$ $0.098 \mathrm{~mm}$. They are obtained with the Varian Unity INOVA, 4.7 Tesla scanner for experimental purpose. The imaging sequence is Spin Echo with TE $=19 \mathrm{msec}$ and $\mathrm{TR}=500 \mathrm{msec}$. An MR-compatible optical force sensor is employed for the reaction force measurement. The rated force and the accuracy of this sensor is $60 \mathrm{~N}$ and $1.0 \%$, respectively. As can be seen in Fig. 3, the reaction force as well as the deformation of the subsurface structure are clearly obtained.

The bottom face of the phantom is glued to the base, and the indenters have the same profile along the longitudinal direction. Mechanical behavior of the center section is thus given by a two-dimensional plane strain model.

\subsection{Estimation Results}

Coarse and fine FE models consist of 133 and 1870 plane strain triangular elements are prepared for the estimation. The same boundary conditions as the MR compression test with the flat indenter are given to these models. Nodal displacements are measured at the boundary of the layers (square-marked 6 points in the first column of Fig. 3. (a)) by manual operations. Since there are 4 successive im-

Table 1. Result of the material constants estimation

\begin{tabular}{|c|c|c|c|}
\hline & \multirow{2}{*}{$\begin{array}{c}\text { Uni-axial } \\
\text { compression test }\end{array}$} & \multicolumn{2}{|c|}{ Our method } \\
\hline & & \begin{tabular}{|l|} 
Coarse model \\
\end{tabular} & Fine model \\
\hline$\mu_{1 s t}$ & $0.0212 \mathrm{~N} / \mathrm{mm}^{2}$ & $0.0229 \mathrm{~N} / \mathrm{mm}^{2}$ & $0.0227 \mathrm{~N} / \mathrm{mm}^{2}$ \\
\hline Error in $\mu_{1 s t}$ & & $8.0 \%$ & $7.1 \%$ \\
\hline$\mu_{2 n d}$ & $0.0356 \mathrm{~N} / \mathrm{mm}^{2}$ & $0.0447 \mathrm{~N} / \mathrm{mm}^{2}$ & $0.0405 \mathrm{~N} / \mathrm{mm}^{2}$ \\
\hline Error in $\mu_{2 n d}$ & - & $25.6 \%$ & $13.8 \%$ \\
\hline$\mu_{3 r d}$ & $0.0654 \mathrm{~N} / \mathrm{mm}^{2}$ & $0.0370 \mathrm{~N} / \mathrm{mm}^{2}$ & $0.0593 \mathrm{~N} / \mathrm{mm}^{2}$ \\
\hline Error in $\mu_{3 r d}$ & - & $43.4 \%$ & $9.3 \%$ \\
\hline$\overline{\text { Error in displacement }(\mathrm{r}}$ & - & $0.17 \mathrm{~mm}$ & $0.17 \mathrm{~mm}$ \\
\hline Error in displacement (max.) & - & $0.34 \mathrm{~mm}$ & $0.38 \mathrm{~mm}$ \\
\hline
\end{tabular}

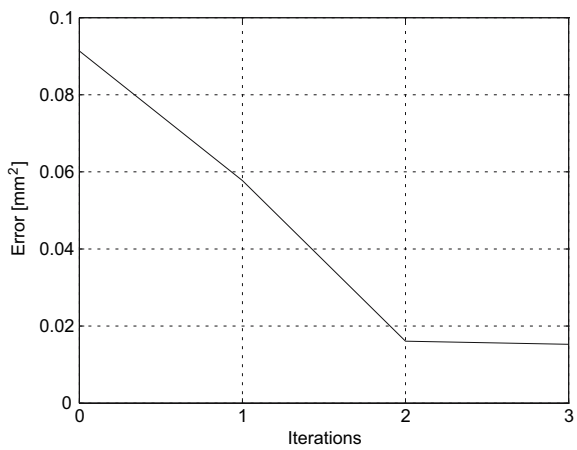

Fig. 4. Transition of the disparity function during the iteration (fine model)

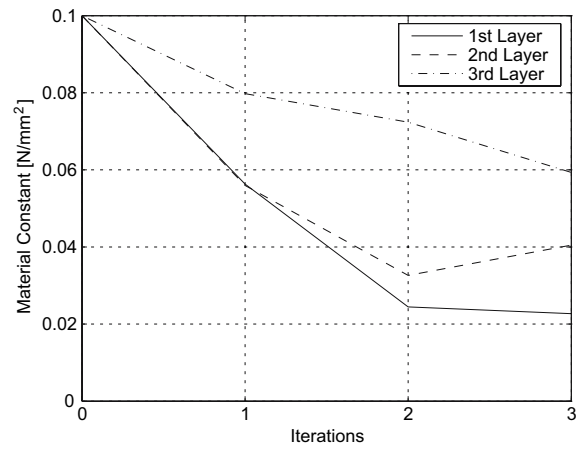

Fig. 5. Transition of the material constants during the iteration (fine model) 


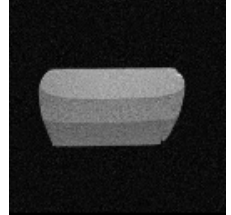

(a) MR image

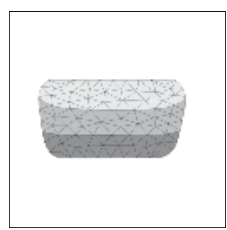

(b) Coarse

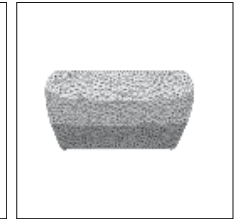

(c) Fine

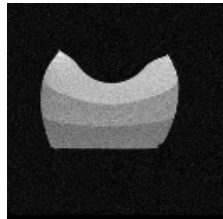

(a) MR image

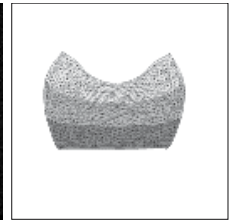

(b) FE analysis

Fig. 6. Result of the iterative FEM analysis

ages (the second to the fifth column of Fig. 3-(a)), total 24 displacement data are used for the estimation of material properties. The material constant $\boldsymbol{m}$ is initially set to $(0.1,0.1,0.1)^{T}$. Note that the initial values are about 1.5 to 4.0 times greater than the material constant identified by the uni-axial compression test.

The iterative FE analysis terminate after 4 iterations with the coarse model and 3 iterations with the fine model. Transition of the disparity function and the material constants are shown in Fig. 4 and Fig. [5 respectively. The results of the estimations are shown in the third and the fourth column of Table 1. It should be remarked that the errors in the material constants estimated with the coarse model are greater than that with the fine model, whereas the errors in the displacement are almost identical in both models.

Figure 6 shows the observed MR image and the result of the FE analysis with the final estimates. There are better correspondences in the whole profile of the phantom between Fig. 6. (a) and (c), while there are correspondences only in the reference points between (a) and (b). This is the causal explanation of the phenomenon described in the previous paragraph. These results suggest that better estimates can be achieved, 1) if the FE model is finer, and 2) if there are enough reference points to be compared even in the coarse model.

\subsection{Reproducibility Test}

In order to validate the estimated material constants, FE simulation of the compression test with a cylindrical indenter is carried out. Figure 7 shows the observed MR image and the result of the simulation with the estimated material constants in the fourth column of Table 1. Better correspondences in the whole profile of the phantom can be confirmed. The mean and the maximum error in the displacement reproducibility are $0.22 \mathrm{~mm}$ and $0.35 \mathrm{~mm}$ that are almost identical to the results in Section 4.3 .

The results of these experiments show the capability of our method that can incorporate both non-linear characteristics and subsurface layered structure of soft tissue. As mentioned in the previous section, the accuracy of the estimation can be improved by using finer model with enough reference points.

\section{Conclusion}

A new estimation method of material properties was presented in this paper. Since this method employs MR observation and iterative FE simulation, it 
can incorporate both non-linear material characteristics and hypodermic layered structure of living soft tissue. The excellent performance of our method was shown by carrying out estimation for a 3-layered silicon rubber phantom. This warrants future works on the noninvasive material properties estimation of real soft tissue.

\section{References}

1. J.F.M.Manschot, A.J.M.Brakkee: The measurement and modeling of the mechanical properties of human skin in vivo-i. the measurement. Journal of Biomechanics 19 (1986) 511-515

2. A.Z.Hajian, R.D.Howe: Identification of the mechanical impedance at the human finger tip. Journal of Biomechanical Engineering 119 (1997) 109-114

3. R.Muthupillai, D.J.Lomas, P.J.A., R.L.Ehman: Magnetic resonance elastography by direct visualization of propagation acoustic strain waves. Science 269 (1995) $1854-1857$

4. J.Bishop, G.Poole, M., D.B.Plewes: Magnetic resonance imaging of shear wave propagation in excised tissue. Journal of Magnetic Resonance Imaging 8 (1998) $1257-1265$

5. T.L.Chenevert, A.R.Skovoroda, M., S.Y.Emelianov: Elasticity reconstructive imaging by means of stimulated echo mri. Magnetic Resonance in Medicine 39 (1998) 382-490

6. G.Soza, R.Grosso, C.G., P.Hastreiter: Estimating mechanical brain tissue properties with simulation and registration. In: Proceeding of the 7th International Conference on Medical Image Computing and Computer Assisted Intervention. Volume 2. (2004) 276-283

7. M.Tada, T.Kanade: An MR-compatible optical force sensor for human function modeling. In: Proceeding of the 7th International Conference on Medical Image Computing and Computer Assisted Intervention. Volume 2. (2004) 129-136

8. R.W.Ogden: Large deformation isotropic elasticity - on the correlation of the theory and experiment for incompressible rubberlike solids. In: Proceedings of the Royal Society of London. (1972) 567-583

9. J.Z.Wu, R.G.Dong, W., A.W.Schopper: Modeling of time-dependent force response of fingertip to dynamic loading. Journal of Biomechanics 36 (2003) 383-392

10. O.H.Yeoh: On the ogden strain-energy function. Rubber Chemistry and Technology 70 (1996) 175-182 